\title{
New working methods for nuclear data evaluation: how to make a nuclear data library?
}

\author{
A.J. Koning ${ }^{\mathrm{a}}$ \\ Nuclear Research and Consultancy Group NRG, P.O. Box 25, 1755 ZG Petten, The Netherlands
}

\begin{abstract}
A call for more modern nuclear data evaluation methodology is made. As an example of the current computational possibilities, nuclear model generated covariances are presented.
\end{abstract}

\section{Introduction}

The strong increase in computer speed and memory has had a significant effect on data evaluation. First of all, nuclear data evaluation relies much more on nuclear model calculations, which can now yield very complete data libraries. Sophisticated reaction theories have become amenable for implementation and even large-scale production of covariance data is now within reach. Also, automated processing scripts and versatile plotting software allows much quicker convergence to the best possible results. In spite of this evolution, nuclear data evaluation methodology has not changed as much as it should have. We still face the situation that all current nuclear reaction data libraries in the world are inconsistent in terms of contents and quality. This has to do with the commonly adopted incremental approach of nuclear data evaluation: improvements to nuclear data libraries are usually performed on a nucleus-by-nucleus or even channel-by-channel basis, driven by a particular evaluation request. Moreover, a material is often evaluated using a certain version of a certain model code, using certain experimental data, at a certain time, filling only certain parts of the data file, by a certain evaluator. Therefore, often isotope A has, e.g., covariance data, but only isotopic angular distributions and no gamma-production data, whereas isotope B has the latter, but very old resonance data, etc. All this leads to a collection of nuclear data files which are partially complete, which originate from different eras and of which the quality is only known to the evaluator (who, especially in this branch of nuclear science, may be retired). The final product is well defined (the ENDF/B-VII, JENDL-3.3, JEFF-3.1, etc. library), but knowledge about the underlying process to create it is scattered over people and time. This poses problems when the library needs to be updated with new experimental or theoretical information. Very precise data, in line with a required target accuracy, may be added to a data file, but if the rest of that evaluated file is in bad shape (e.g., isotropic angular distributions), the impact of such a highprecision measurement will be reduced.

\footnotetext{
${ }^{a}$ Presenting author, e-mail: koning@nrg-nl.com
}

Instead of such an incremental ad hoc approach, a working method should be adopted that is more in line with the quality assurance procedures that are more and more required by our technological and industrial partners. Not only will the result be more easily and better validated, but we are also doing ourselves a favor, since manual changes may induce errors (or just because we want to be efficient), if we can easily reproduce the entire evaluation process in case new information emerges (new measurements, a discovered bug in the model code, etc.). The challenge is to implement this, even though at first this may seem not very challenging from a scientific point of view, rather than attacking the next experimental or theoretical nuclear physics problem. It will yield a nuclear data library whose contents are on par with the existing experimental data and the current status of nuclear model codes. In other words, the current knowledge of nuclear reaction physics would actually be present in the data library. Obviously, the final objective of all this is to produce a highquality nuclear data library. But how is the "quality" of a nuclear data library defined? In the entire nuclear science community that revolves around nuclear data libraries, there are at least the following different interpretations:

1. User satisfaction: if a processed nuclear data library and the associated reactor physics software perform well in a production environment, such as nuclear power plants, the customer is satisfied. If the reactor engineer can safely interpolate the calculated results between the cases where the file is post-adjusted to (often proprietary) reactor experiments, there may be no incentive to upgrade to a more modern data file, since that requires costly re-adjustment of all engineering factors. The improved physical content of the data file, as advocated by the evaluator, is then not always relevant. In other words, a very strong case for adopting a more modern nuclear data file is always needed.

2. Reproduction of integral measurements: various criticality, shielding, and reactor experiments have been performed in the past. Fortunately, an increasing number of them is now becoming available in well defined benchmark collections, such as ICSBEP and IRPHE. If possible, a new evaluation for a material should be tested against as many integral experiments as possible, using as many different reactor 
codes as possible. Reproduction of the integral data then reveals the quality of the data file.

3. Completeness: a nuclear data file should include a complete resonance data set, all cross sections, angular distributions, double-differential spectra, gamma production, recoils, isomeric ratios and covariances, as well as fission related data for all actinides.

4. Correctness and processibility (NJOY-able): the format of a nuclear data file should exactly follow the ENDF6 format recommendations and should successfully pass format-checking codes. The structure of the data file should be such that flawless processing, usually by the NJOY code, is guaranteed for both multi-group and pointwise user libraries.

5. Representation of differential measurements: confidence in a nuclear data file will be larger when the tabulated data correspond with high quality microscopic measurements.

6. Underlying physics that produces data file: since there exists only a very finite set of experimental data, nuclear models need to be used to interpolate between measurements and extrapolate beyond them. Faith in the quality of the nuclear data file is related to the status of the physics that is implemented in the model codes.

These are quality definitions from commercial reactor engineers, reactor physicists, nuclear data evaluators, data processors and data centers, experimental nuclear physicists and theoretical nuclear physicists, respectively. The ideal situation would be that all 6 requirements are simultaneously satisfied by one and the same data file, but this is seldom (probably never) the case. Nevertheless, we argue that items 2-6 should be cast into one evaluation/validation scheme. Especially for innovative designs, which rely on extrapolation rather than interpolation from well-known cases, a secure well-validated approach is crucial. Figures 1 and 2 summarize this for data evaluation and validation, respectively. Once the nuclear model code and ENDF formatting code used in the evaluation process is well verified and validated, the 4 boxes labeled "Save" contain the essential information that produced the nuclear data file. All relevant experimental data for the nucleus under consideration should be readily available, as well as a file with deduced resonance parameters. An input file for the nuclear model code with parameters adjusted to reproduce the available experimental data produces a complete set of nuclear reaction results. Finally, a formatting code produces the ENDF-6 data file, which is driven by a script that performs any additional actions such as copy-paste from existing data libraries or scientifically dubious adjustments for the sake of good performance of the data file in applications. As figure 1 suggests, this could be done for all nuclides and the system would consist of "blind" evaluations for isotopes which are either relatively unimportant or have no experimental data available, and very detailed evaluations with fine tuned model parameters, direct inclusion of certain sets of experimental data, etc. The central message is that the data library can be automatically produced from its components, and that the knowledge of the data evaluator can be preserved in a much more compact way than in an entire ENDF-6 data file.

For a certain class of validation, criticality and shielding calculations with a Monte Carlo approach, extensive schemes

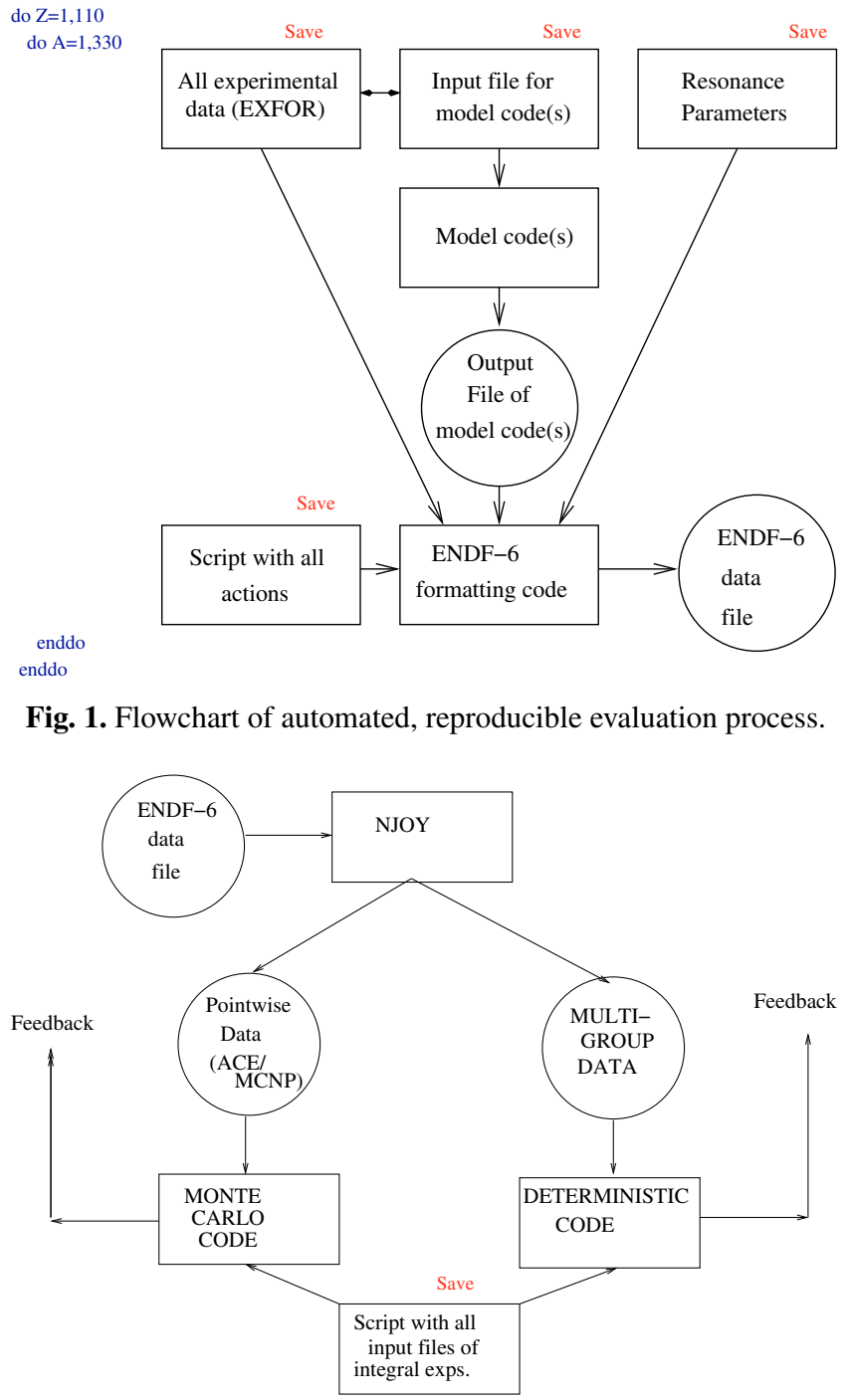

Fig. 2. Flowchart of automated, reproducible validation process.

already exist [1]. Validation should give direct feedback to the data evaluation process and the evaluation and validation schemes become basically linked, see also ref. [2]. No scientific breakthrough is required to push the current nuclear data libraries to this higher level, it only requires a more modern, quality-assured working method. Of course, before we can proceed several boundary conditions need to be met. Modern nuclear data evaluation requires:

- An unambiguous, well-established experimental database. All data in EXFOR need to be computationally available and the most flagrant format errors need to be corrected. A new WPEC subgroup, SG30, has been launched to accomplish this.

- Verified and validated nuclear model codes in combination with a nuclear model parameter database (RIPL).

- A quality assured evaluation procedure that guarantees complete reproducibility of the results that fill the data files, i.e., automatic file production on the basis of selected experimental data and working input files, with optimized parameters, for model codes. This should be pursued even if many actions were needed for individual reaction 
channels e.g., direct inclusion of experimental data, or ad hoc modifications to particular reaction channels.

The reward of such an approach will be a new generation of nuclear data libraries, with the following attractive features:

- Isotopic evaluations that are mutually equivalent in terms of completeness, underlying physics and ENDF format, i.e., we use the same ENDF procedure for each isotope. All evaluations extend to an energy of $200 \mathrm{MeV}$.

- Data files whose contents are on par with the existing experimental data and the current status of nuclear model codes. Also, this allows a very short delay between the release of new experimental data and nuclear model updates and their use to update nuclear data files.

- A system that allows for an easy transition to a modern format, whenever the data format, processing, and reactor physics communities and our customers are ready for that.

If we are certain that we have produced the best possible, complete data library to date, using all available experimental and theoretical information, i.e., that we can not deliver our data better than specified by our covariance matrix, we have a strong case for new high-precision measurements and theory development for new applications, such as GEN-IV reactors, if their target accuracies are still tighter than we can give.

\section{Random covariance model}

An essential ingredient of the scheme outlined above is covariance data. Therefore, we have chosen covariances as the technical contribution to this paper. The assessment of the uncertainties in nuclear reaction data is important for both basic physics and technological applications. First, it defines the current quality of both experimental and theoretical nuclear reaction physics. Experimental uncertainties represent a sort of limit of the current-day precision with which we can measure a particular observable (the fact that these uncertainties have a systematical and a statistical component requires a discussion on its own). Theoretical uncertainties would represent the extent to which we are (un)able to cast nature into models and their associated parameters. Second, nuclear reaction data, such as cross sections, resonance parameters, energy spectra and angular distributions are of prime importance to the computational simulation of nuclear devices. A reliable assessment of the uncertainties in calculated integral reactor parameters depends directly on the uncertainties of the underlying nuclear data. Starting from the covariance matrices of the basic nuclear data, error propagation in transport, reactor, activation, etc. codes enables to estimate the uncertainties of calculated design parameters, which has a profound impact on issues of general concern such as safety and economy.

An often heard complaint is that covariance nuclear data are scarce, not only because a significant experimental database for the isotope under consideration must be available, but also because the covariance evaluation process can be rather complex and time-consuming. One of the reasons for this situation is that, while the good habit of delivering error bars is followed by almost all experimental nuclear physicists, theoreticians seldom provide uncertainties, let alone a full covariance matrix, with their results. With the help of a flexible nuclear model code and the current-day computer power, it is now possible to attack these problems. We note that the basic ideas of this approach have already been outlined by Smith [3], and we apply it here to show that the method is actually feasible. For all our calculations we use the TALYS code [4].

A short, simple mathematical formalism will suffice to explain the method of covariance generation from random model parameters. Often, a nuclear model calculation is performed with various model parameters $p_{1}, p_{2}, \ldots$, of which some are usually adjusted to obtain the optimal description of experimental data. An input file may for example contain the optical model parameters $p_{1}=r_{v}$ (real central radius), $p_{2}=a_{v}$ (real central diffuseness), etc., followed by the level density parameters and so on. Let $\mathbf{p}$ be the vector of the $L$ adjustable nuclear model parameters that are relevant to the problem under consideration, i.e.,

$$
\mathbf{p}=\left\{p_{1}, \ldots, p_{l}, \ldots, p_{L}\right\}
$$

In practice $L$ may take on values from one up to several tens. The case of many adjustable parameters occurs, e.g., for complete optical model studies or when many residual nuclides, each characterized by its own parameters (level density, etc.), are involved in a calculation. Next, let $\sigma$ be a vector of $N$ calculated quantities,

$$
\boldsymbol{\sigma}=\left\{\sigma_{1}, \ldots, \sigma_{i}, \ldots, \sigma_{N}\right\}
$$

The obvious choice for the elements of $\sigma$ are cross sections, and we will indeed generally use the term "cross sections" for $\sigma$. It should however be understood that $\sigma$ may contain all other quantities of interest as well, such as angular distributions, differential energy spectra, etc. For example, for a statistical study on parameters of the optical model and the associated observables, $\sigma$ may contain total cross sections $\sigma^{\text {tot }}$ and elastic angular distributions $d \sigma^{\mathrm{el}} / d \Omega$ at various incident energies and outgoing angles. As another example, if we are interested in a covariance matrix for applied purposes (evaluated nuclear data file), $\sigma$ may contain the total and partial cross sections for all open reaction channels at various incident energies. Whatever the objective, it is obvious that $\sigma$ may contain a very large number of elements. The vector $\sigma$ is a function $T$ of the vector $\mathbf{p}$,

$$
\sigma=T(\mathbf{p})
$$

where in this work $T$ stands for the entire TALYS nuclear model code. The basis of our method is to let TALYS perform many calculations, whereby each time all $L$ elements of the $\mathbf{p}$ vector are randomly sampled from a normal distribution with a specific width $\Delta p_{l}$ for each parameter $p_{l}$, i.e.,

$$
p_{l}^{(k)}=p_{l}^{(0)} \pm \Delta p_{l}, \quad l=1, L,
$$

where we have added a superscript $(k)$ to denote the $k$-th TALYS run, i.e.,

$$
\boldsymbol{\sigma}^{(k)}=T\left(\mathbf{p}^{(k)}\right) .
$$

The initial set of input parameters, the central values, is denoted as $\mathbf{p}^{(0)}$ and the corresponding initial calculated cross 
section vector as $\boldsymbol{\sigma}^{(0)}$, obtained from the final TALYS calculation. This initial set represents generally one of the following two cases: (a) the best possible parameter set, adjusted to experimental data, obtained from a numerical and/or visual optimization procedure, or (b) a so-called global nuclear model calculation with all parameters equal to their default values. After the initial calculation, the first set of random parameters $\mathbf{p}^{(1)}$ will lead to a set of cross sections $\boldsymbol{\sigma}^{(1)}$ that is different from $\boldsymbol{\sigma}^{(0)}$. From the results, we can obtain the single covariance matrix element $\left(\sigma_{i}^{(1)}-\sigma_{i}^{(0)}\right)\left(\sigma_{j}^{(1)}-\sigma_{j}^{(0)}\right)$ which is a measure of the change of $\sigma_{j}$ relative to the change of $\sigma_{i}$. In a Monte Carlo approach, such a single covariance matrix element alone is rather meaningless, since all parameters are simultaneously sampled from a random distribution, and no significant information is available after one run. However, after performing many calculations, all statistical information like average values, uncertainties and covariance matrices will gradually become available. The average covariance matrix for cross sections is given by

$$
V_{i j}=\frac{1}{K} \sum_{k=1}^{K}\left(\sigma_{i}^{(k)}-\sigma_{i}^{(0)}\right)\left(\sigma_{j}^{(k)}-\sigma_{j}^{(0)}\right), \quad i, j=1, N
$$

where $K$ is the total number of TALYS runs needed for statistical convergence. Similarly, the average relative covariance matrix for cross sections can be obtained,

$$
R_{i j}=V_{i j} /\left(\sigma_{i}^{(0)} \sigma_{j}^{(0)}\right), \quad i, j=1, N .
$$

The extent to which the calculated results co-vary is most clearly represented by the average correlation matrix for cross sections

$$
C_{i j}=V_{i j} / \sqrt{V_{i i} V_{j j}}, \quad i, j=1, N,
$$

whose elements take on values between -1 (complete anticorrelation), 0 (no correlation) and 1 (complete correlation). As an example, if by changing model parameters the total neutron non-elastic cross section at a certain incident energy remains equal, while the $(n, 2 n)$ cross section increases, we expect the $(n, n$ ') cross section to decrease, and this anticorrelation will be reflected by a negative value of the $C_{i j}$ matrix element that relates $\sigma_{n, n^{\prime}}$ and $\sigma_{n, 2 n}$. The average calculated cross sections are

$$
\bar{\sigma}_{i}=\frac{1}{K} \sum_{k=1}^{K} \sigma_{i}^{(k)}, \quad i=1, N
$$

for which one would expect that $\bar{\sigma}_{i} \approx \sigma_{i}^{(0)}$ for all elements $i$. The square root of the diagonal elements of the relative covariance matrix $R$ represents the uncertainty. Hence, the final calculated cross sections together with their uncertainties can be expressed as

$$
\sigma_{i}^{\mathrm{final}}=\bar{\sigma}_{i}\left(1 \pm \sqrt{R_{i i}}\right), \quad i=1, N
$$

Actually, the values of equations (6)-(10) can be updated, and inspected, after every TALYS run, and statistical convergence is reached when the running index $k$ approaches $K$ (for which an appropriate value needs to be determined).
We can construct similar statistical quantities for the $L$ model parameters that we use in every run, such as the average covariance matrix for model parameters,

$$
P_{l m}=\frac{1}{K} \sum_{k=1}^{K}\left(p_{l}^{(k)}-p_{l}^{(0)}\right)\left(p_{m}^{(k)}-p_{m}^{(0)}\right), \quad l, m=1, L,
$$

the relative covariance matrix for model parameters,

$$
R_{l m}^{P}=P_{l m} /\left(p_{l}^{(0)} p_{m}^{(0)}\right), \quad l, m=1, L,
$$

and the correlation matrix for model parameters,

$$
C_{l m}^{P}=P_{l m} / \sqrt{P_{l l} P_{m m}}, \quad l, m=1, L .
$$

To complete the analogy with the statistical information for the cross sections, the average model parameters are,

$$
\bar{p}_{l}=\frac{1}{K} \sum_{k=1}^{K} p_{l}^{(k)}, \quad l=1, L
$$

where we should obtain $\bar{p}_{l} \approx p_{l}^{(0)}$. The final model parameters with their uncertainties are

$$
p_{l}^{\text {final }}=\bar{p}_{l}\left(1 \pm \sqrt{R_{l l}^{P}}\right), \quad l=1, L
$$

Of course, equations (11)-(15) are meaningless when we take uncorrelated, randomly sampled parameters as starting point: after enough iterations, we will simply obtain as a result what we put in, provided the random generator is reliable. The parameter covariance matrix will be, $P_{l m}=0$ for $l \neq m$, since we assume to have no a priori knowledge of the correlation of the model parameters. Also, equation (15) becomes equal to equation (4). The statistical expressions for the model parameters do however become meaningful when we introduce parameter correlations. There exist various mathematical rigorous methods for automatic parameter/cross section optimization, mostly based on Bayesian statistics. These methods include experimental data, with or without proper covariance matrices, directly in the optimization procedure, which yields both a parameter correlation matrix and a set of the optimized cross sections [5]. Alternatively, correlated sampling using multivariate Gaussian distributions could be performed, if one wishes to pursue Monte Carlo covariance methods. Similar techniques are used in the backward-forward Monte Carlo methods [6]. In theory, both methods are correct, but often, the experimental data needs to be "pre-conditioned" to avoid unrealistic results. In this work, we take the point of view that mathematical rigour is welcome once both the experimental uncertainties and the uncertainties in the theoretical nuclear models is well under control. Until this has been accomplished, we are in some sense less ambitious: as a first step, we find it safer to use the collection of experimental data as a visual guide to obtain realistic results, i.e., we do not literally include them in the optimization scheme. We are however ambitious in the final objective, namely to establish a universal set of nuclear model parameter uncertainties that can safely be used as realistic prior in covariance studies. Once this is established, one can zoom in on a particular nuclide, use its available experimental data, possibly make use 
Table 1. Potential depth parameters and Fermi energy for the neutron global OMP of equation (17).

\begin{tabular}{|c|c|}
\hline $\begin{array}{l}v_{1}^{n}=59.30-21.0(N-Z) / A-0.024 A \\
v_{2}^{n}=0.007228-1.48 .10^{-6} A \\
v_{3}^{n}=1.994 .10^{-5}-2.0 .10^{-8} A \\
v_{4}^{n}=7 \cdot 10^{-9} \\
w_{1}^{n}=12.195+0.0167 A \\
w_{2}^{n}=73.55+0.0795 A \\
d_{1}^{n}=16.0-16.0(N-Z) / A \\
d_{2}^{n}=0.0180+0.003802 /(1+\exp [(A-156 .) / 8 .]) \\
d_{3}^{n}=11.5 \\
v_{s o 1}^{n}=5.922+0.0030 A \\
v_{s o 2}^{n}=0.0040 \\
w_{s o 1}^{n}=-3.1 \\
w_{s o 2}^{n}=160 . \\
E_{f}^{n}=-11.2814+0.02646 A\end{array}$ & $\begin{array}{l}\mathrm{MeV}^{-1} \\
\mathrm{MeV}^{-2} \\
\mathrm{MeV}^{-3} \\
\mathrm{MeV} \\
\mathrm{MeV} \\
\mathrm{MeV} \\
\mathrm{MeV}^{-1} \\
\mathrm{MeV}^{\mathrm{MeV}} \\
\mathrm{MeV}^{-1} \\
\mathrm{MeV} \\
\mathrm{MeV} \\
\mathrm{MeV}\end{array}$ \\
\hline
\end{tabular}

of the optimization methods mentioned above and demand that the final results do not deviate completely from the universal set. To obtain the final cross section uncertainties and the parameter correlations, we use a simple binary reject/accept method: we put an uncertainty band around the best, or global, data set $\sigma^{(0)}$, such that the available scattered experimental data falls more or less inside this uncertainty band. Next we make sure that our initial parameter uncertainties produce cross section uncertainty bands that are somewhat larger than those indicated by the experimental data. If the calculated cross sections fall inside the experimentally determined uncertainty band we accept the input parameter set, otherwise we reject it. We generally aim for an accept/reject ratio of 1:2. After enough iterations, a full parameter covariance matrix is obtained, which now includes off-diagonal correlations since only certain combinations of nuclear model parameters led to results that were accepted. This procedure can be followed for one particular reaction channel or for all open channels simultaneously.

Finally, we can relate the variation of the cross sections to the variation of the model parameters. This can be done through the average relative sensitivity matrix $S$, which is defined as

$S_{i l}=\frac{\sum_{k=1}^{K}\left(p_{l}^{(k)}-p_{l}^{(0)}\right)\left(\sigma_{i}^{(k)}-\sigma_{i}^{(0)}\right)}{\sum_{k=1}^{K}\left(p_{l}^{(k)}-p_{l}^{(0)}\right)^{2}} \frac{p_{l}^{(0)}}{\sigma_{i}^{(0)}}, \quad i=1, N, \quad l=1, L$.

which sheds light on the relative importance of the model parameters. $S$ is dimensionless and directly reflects the dependence of a cross section $\sigma_{i}$ on the model parameter $p_{l}$. For example, a value of -3.5 for the element $S_{i l}$ means that if the model parameter $p_{l}$ is increased by $10 \%$, the cross section $\sigma_{i}$ decreases by $35 \%$. Again, $S$ will converge to its final value after many calculations. Actually, it is the statistical convergence of $S$ that determines the appropriate value for $K$. One advantage of the Monte Carlo approach is that each $\sigma_{i}$ generally does not depend on all parameters. This means that in one and the same run we contribute to the statistical convergence of uncorrelated $\sigma_{i}$ simultaneously. If a particular cross section depends on only 1 or 2 parameters, then the average of the associated covariance matrix elements will be obtained quicker than for a cross section that depends on many parameters. This information is contained in $S$. We thus have to wait for the convergence of the cross section that depends on the most parameters, until we can adopt equations (6)-(15) as the final results.

\section{Uncertainties of optical model parameters}

In the quest for a universal set of uncertainties for nuclear model parameters, assessing the uncertainty of optical model potential (OMP) parameters is a natural first step. Therefore, we first investigate to what extent out Monte Carlo method can reproduce the deviation between our global OMP [7] and experimental data. To make this paper self-contained, we repeat the form of the global neutron OMP from ref. [7],
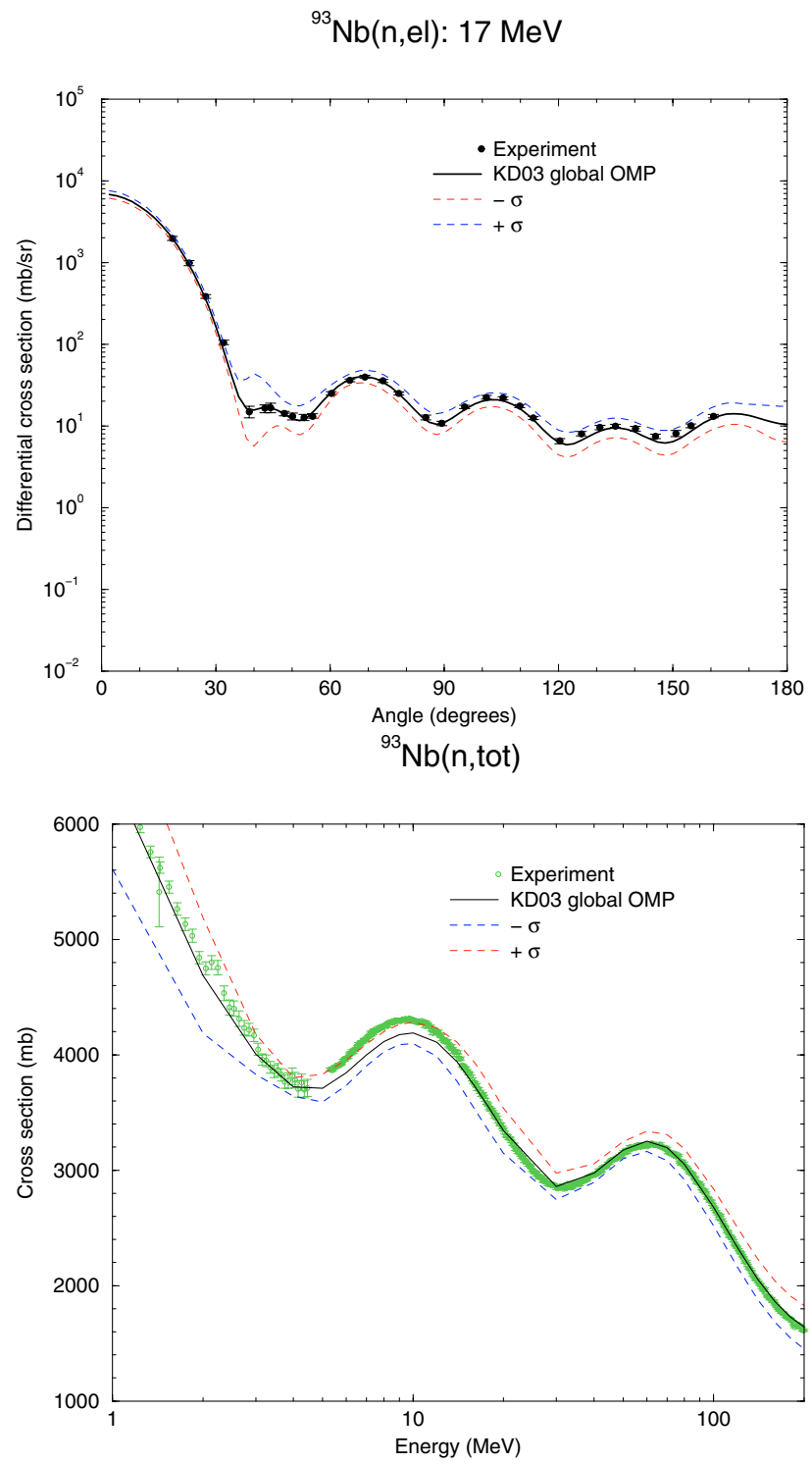

Fig. 3. Comparison of experimental angular distributions (top) and total cross sections (bottom) and calculated results including uncertainty for $\mathrm{n}+{ }^{93} \mathrm{Nb}$. 
validated for $0.001 \leq E \leq 200 \mathrm{MeV}$ and $24 \leq A \leq 209$,

$$
\begin{aligned}
& V_{V}(E)=v_{1}^{n}\left[1-v_{2}^{n}\left(E-E_{f}^{n}\right)+v_{3}^{n}\left(E-E_{f}^{n}\right)^{2}-v_{4}^{n}\left(E-E_{f}^{n}\right)^{3}\right] \\
& W_{V}(E)=w_{1}^{n} \frac{\left(E-E_{f}^{n}\right)^{2}}{\left(E-E_{f}^{n}\right)^{2}+\left(w_{2}^{n}\right)^{2}} \\
& r_{V}=1.3039-0.4054 A^{-1 / 3}, a_{V}=0.6778-1.487 \cdot 10^{-4} A \\
& W_{D}(E)=d_{1}^{n} \frac{\left(E-E_{f}^{n}\right)^{2}}{\left(E-E_{f}^{n}\right)^{2}+\left(d_{3}^{n}\right)^{2}} \exp \left[-d_{2}^{n}\left(E-E_{f}^{n}\right)\right] \\
& r_{D}=1.3424-0.01585 A^{1 / 3}, a_{D}=0.5446-1.656 \cdot 10^{-4} A \\
& V_{S O}(E)=v_{s o 1}^{n} \exp \left[-v_{s o 2}^{n}\left(E-E_{f}^{n}\right)\right] \\
& W_{S O}(E)=w_{s o 1}^{n} \frac{\left(E-E_{f}^{n}\right)^{2}}{\left(E-E_{f}^{n}\right)^{2}+\left(w_{s o 2}^{n}\right)^{2}} \\
& r_{S O}=1.1854-0.647 A^{-1 / 3}, \quad a_{S O}=0.59,
\end{aligned}
$$

where $E$ is the incident energy in the LAB system, the units are in $\mathrm{fm}$ and $\mathrm{MeV}$ and the parameters are given in table 1 . We have tested our Monte Carlo method for several nuclides and a selection of the results is shown in figure 3. TALYS has been set in a mode in which only the optical model related observables are calculated, and 1000 runs with random optical model parameters have been performed. In line with the approach outlined in the previous section, we have adjusted the widths of the model parameters, see equation (4), such that we simultaneously get reasonable agreement for a subset of our large experimental OMP database of ref. [7]. The resulting uncertainties of the global neutron OMP parameters are given in table 2. We emphasize that for these results we have not yet used the accept/reject method: the a priori uncertainties of table 2 give the results displayed in the figures. Of course, we can not display all results, but we can report that on average, the uncertainty bands are large enough to cover the deviation between the global results and experimental data. For a few cases we are slightly too optimistic, and sometimes we are a bit too conservative, e.g., for total cross sections above $20 \mathrm{MeV}$. More definite answers can be expected when we include more experimental data and apply the binary accept/reject method outlined in the previous section. This method can directly be applied to all non-elastic channels and their associated nuclear model parameters such as total and partial level density parameters, fission barrier parameters, gamma-ray strength functions and pre-equilibrium parameters.

Table 2. Uncertainties of the global neutron OMP parameters, given as fraction $(\%)$ of the absolute value.

\begin{tabular}{llll}
\hline Parameter & uncertainty (\%) & Parameter & uncertainty (\%) \\
\hline$r_{V}$ & 2 & $d_{1}^{n}$ & 10 \\
$a_{V}$ & 2 & $d_{2}^{n}$ & 10 \\
$v_{1}^{n}$ & 2 & $d_{3}^{n}$ & 10 \\
$v_{2}^{n}$ & 3 & $r_{S O}$ & 10 \\
$v_{3}^{n}$ & 3 & $a_{S O}$ & 10 \\
$v_{4}^{n}$ & 5 & $v_{s o 1}^{n}$ & 5 \\
$w_{1}^{n}$ & 10 & $v_{s o 2}^{n}$ & 10 \\
$w_{2}^{n}$ & 10 & $w_{s o 1}^{n}$ & 20 \\
$r_{D}$ & 3 & $w_{s o 2}^{n}$ & 20 \\
$a_{D}$ & 4 & & \\
\hline
\end{tabular}

\section{Conclusions}

A breakthrough in nuclear data evaluation methodology is advocated, without the immediate necessity of a breakthrough in experimental or theoretical nuclear physics. This is possible, simply because the current status of experimental and theoretical nuclear physics has not yet been systematically processed into the nuclear data libraries for applications. An automated evaluation scheme is proposed, which brings all nuclear data files on par with the existing experimental data and the current power of nuclear model codes, and in which new measurements and theoretical developments can immediately be processed into an updated datafile. Of course, this plan does not take away the necessity to break through certain scientific barriers, both experimental and theoretical, which is needed to reach certain target accuracies of innovative designs (GEN-IV, transmutation). Especially for actinides, current fission models are not yet of a quality that guarantees realistic results in the absence of experimental data. To prove that the method outlined here is successful, one could start modestly and perform this for all non-fissile nuclides and produce data libraries that include the best resonance data, all cross sections, angular distributions, double-differential spectra, gamma production, recoils, isomeric ratios and covariances. Direct, and significant impact can be expected for fusion (ITER and IFMIF), fission products, structural materials and coolants. Conceptually, there is however nothing that prevents an extension of this approach to actinides. This evaluation scheme can be combined with an automated validation scheme (for this we need to bring all existing integral experiments under ICSBEP regime) which provides direct feedback to the evaluation process. This approach can only be successful if we clean up and correct the EXFOR database, enabling automatic comparison with nuclear model codes for all nuclides/reactions. An essential component to be delivered with all nuclear data files is covariance data, and we have shown in this paper that covariances of reasonable quality can now be produced.

\section{References}

1. S.C. van der Marck, Benchmarking ENDF/B-VII.0, Nucl. Data Sheets 107, 3061 (2006).

2. M. Herman, EMPIRE ultimate expansion: resonances and covariances (these proceedings).

3. D. Smith, Covariance matrices for nuclear cross sections derived from nuclear model calculations, Argonne report ANL/NDM159, November 2004.

4. A.J. Koning, S. Hilaire, M.C. Duijvestijn, TALYS-1.0 (these proceedings).

5. D. Rochman, M. Herman, P. Oblozinsky, S. Mughabghab, T. Kawano, Neutron cross section covariance calculations from thermal energy to $20 \mathrm{MeV}$ (these proceedings).

6. E. Bauge, S. Hilaire, P. Dossantos-Uzarralde, Evaluation of the covariance matrix of neutronic cross sections with the backwardforward Monte Carlo Method (these proceedings).

7. A.J. Koning, J.P. Delaroche, Local and global nucleon optical models from $1 \mathrm{keV}$ to $200 \mathrm{MeV}$, Nucl. Phys. A 713, 231 (2003). 